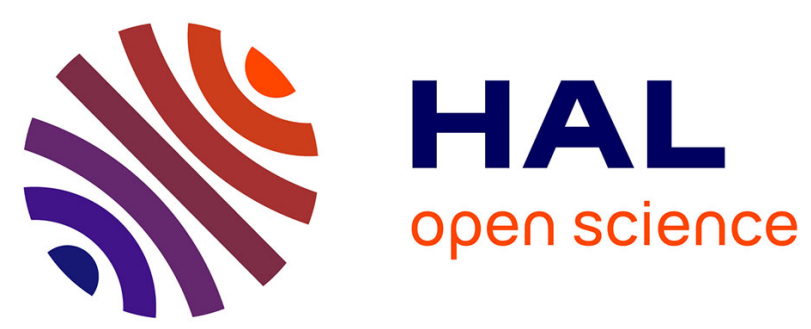

\title{
Associativité, symbolisation et entropie : propositions théoriques et cliniques \\ Thomas Rabeyron
}

\section{To cite this version:}

Thomas Rabeyron. Associativité, symbolisation et entropie: propositions théoriques et cliniques. Annales Médico-Psychologiques, Revue Psychiatrique, 2015, 173 (8), pp.649-658. 10.1016/j.amp.2015.02.009 . hal-02173618

\section{HAL Id: hal-02173618 \\ https://hal.univ-lorraine.fr/hal-02173618}

Submitted on 4 Jul 2019

HAL is a multi-disciplinary open access archive for the deposit and dissemination of scientific research documents, whether they are published or not. The documents may come from teaching and research institutions in France or abroad, or from public or private research centers.
L'archive ouverte pluridisciplinaire HAL, est destinée au dépôt et à la diffusion de documents scientifiques de niveau recherche, publiés ou non, émanant des établissements d'enseignement et de recherche français ou étrangers, des laboratoires publics ou privés. 
Associativité, symbolisation et entropie : propositions théoriques et cliniques

Thomas Rabeyron

Professeur de psychologie clinique et psychopathologie Université de Lorraine (Interpsy ; Psyclip) 


\section{Résumé}

L'association libre est un concept fondateur des thérapies d'inspiration psychanalytique en tant que technique d'exploration de la réalité psychique mais aussi comme méthode thérapeutique. Nous proposons dans ce travail d'étudier ses fondements, ainsi que les logiques de l'associativité qui en découlent, à partir d'un double regard issu de la psychanalyse et des neurosciences cognitives. Dans cette perspective, nous présentons tout d'abord ses origines historiques dans le champ psychanalytique. Les recherches issues de la psychologie cognitive (Daniel Kahneman), des neurosciences cognitives (Karl Friston, Robin Carhart-Harris) et de la neuropsychanalyse (Marks Solms) sont ensuite l'occasion de mieux saisir l'hétérogénéité du fonctionnement psychique et ses modes de symbolisation. L'associativité apparaît alors, à la croisée des modèles, comme un concept fécond qui favorise l'articulation entre psychanalyse et neurosciences à partir, notamment, des concepts d'énergie libre et d'entropie. La notion de transmodalité symbolisante est enfin proposée comme tentative de synthèse des rapports de l'associativité aux différents modes de symbolisation.

Mots clés: Association libre ; Historique; Modèle ; Neurosciences; Psychanalyse; Psychologie sociale 


\section{Associativité, co-associativité et réflexivité}

L'évolution actuelle des pratiques et des dispositifs cliniques nécessite de déterminer de manière approfondie ce qui fonde et caractérise l'efficacité de l'activité psychothérapique. Outre l'évaluation quantitative des prises en charge, une réflexion portant sur les processus nous paraît fondamentale pour comprendre au plus près la pertinence des modèles théoriques et cliniques. Cette capacité à repérer les processus rejoint la mouvance actuelle visant à croiser les données provenant de l'observation clinique, en particulier dans le champ psychanalytique, et les recherches issues des neurosciences cognitives [40] [40], [47].

L'association libre apparaît de ce point de vue comme un concept fécond qui offre l'opportunité d'étudier la qualité et l'évolution des processus psychiques à la croisée des modèles. L'association libre représente en effet la «règle fondamentale» de l'approche psychanalytique, qui implique de demander au sujet de dire spontanément, et sans retenue, tout ce qui lui vient à l'esprit. Le clinicien sera alors attentif à l'associativité, c'est-à-dire, globalement, à la manière dont le sujet passera, avec plus ou moins de fluidité, d'une représentation à une autre. L'associativité peut alors prendre des formes multiples selon les dispositifs : elle sera, par exemple, focale lorsqu'elle est centrée sur un rêve ou un test projectif ; groupale, quand elle découle de la chaine associative impliquant plusieurs sujets ; médiatisée et corporelle si elle externalisée et étayée sur une médiation.

Comprendre les logiques actuelles de l'associativité implique tout d'abord d'en situer les origines historiques. L'activité de pensée, et l'associativité qui la caractérise, est ainsi représentée, au XVIII siècle, de manière «extériorisée », avec Franz-Anton Mesmer et le concept de « magnétisme animal », en tant que « flux universel » dont il conviendrait de rétablir l'harmonie à l'aide de différents procédés (magnétisme, passes, baquet, etc.) [41]. Cette première tentative de représentation du «flux psychique» sera progressivement "intériorisée », par étapes successives, jusqu'au développement de la psychanalyse [52]. Mais les différents « bricolages » qu'ont produit les cliniciens depuis cette époque ont gardé la trace d'une pensée en tant que « flux » qui peut demeurer «bloqué », devenant source de différentes formes de psychopathologies, ce que l'on retrouve dans les théories actuelles de l'associativité [13], [53], [54].

Janet évoquait plus précisément des «points de fixation » dans l'activité psychique et Freud présenta dès l'Esquisse d'une psychologie scientifique, en 1895, l'hypothèse de «défenses primaires » conduisant à ces points de fixation. Dans les théories psychanalytiques, la psychopathologie est ainsi appréhendée selon le prisme de la capacité à associer, selon la « fluidité » du fonctionnement psychique et les particularités du flux associatif. L'originalité de ces modalités défensives comme le résultat de structurations antérieures de la psyché liées à la vie affective et sexuelle. L'architecture même de l'appareil psychique et ses formes originaires, en ce qu'elles contiennent et enveloppent l'activité de pensée, limitent donc ses capacités associatives. Dans Constructions en Analyse [19], Freud remarque que ces fixations proviendraient, dans certains cas, d'un « noyau de réalité historique » qui se répète sur le mode de la compulsion de répétition du fait d'une « faiblesse de la synthèse » du Moi. Freud précise également de quelle manière un « investissement latéral inhibant » permettrait d'éviter le retour de la perception d'événements pénibles qui participe aussi d'un défaut d'associativité.

C'est par la règle de la « libre association » qu'il sera possible d'avoir une action sur les « complexes associatifs » qui tendent à figer l'activité de pensée. Freud commença à dégager les fondements de l'association libre avec Emmy Von N., en 1889, avant de les développer dans La méthode d'interprétation des rêves, en 1900 [20]. Il utilise ensuite cette technique avec Dora [21] avant de véritablement la déployer, en 1907, avec L'homme aux rats [22]. Dans $L a$ technique psychanalytique, en 1913, il propose une métaphore qui permet de saisir plus précisément la manière dont le patient est placé dans un état d'associativité particulier: 
« Comportez-vous à la manière d'un voyageur qui, assis près de la fenêtre de son compartiment, décrirait le paysage tel qu'il se déroule à une personne placée derrière lui. Enfin, n'oubliez jamais votre promesse d'être tout à fait franc, n'omettez rien de ce qui, pour une raison quelconque, vous paraît désagréable à dire » [23] (p. 94). L'association libre consiste ainsi en une technique d'investigation de la réalité psychique et de ses processus inconscients, mais aussi en un procédé aux vertus psychothérapiques, au-delà de sa dimension cathartique. Elle permet de relancer le « flux » d'une associativité restée en panne. Qu'est-ce qui caractérise alors la manière dont l'être humain passe spontanément d'une idée à une autre dans le cadre thérapeutique? Que découvre-t-on par le biais de l'association libre sur le fonctionnement psychique et les troubles psychopathologiques?

Freud relève tout d'abord que l'associativité est liée à une certaine " hétérogénéité 》 des processus psychiques. Il propose ainsi de distinguer registres primaire et secondaire [24], [25]. Ceux-ci correspondent à un «traitement» différent de l'énergie psychique qui engendre une associativité spécifique pour chacun d'entre eux. Dans les premiers modèles freudiens, les processus primaires, du point de vue topique, caractérisent le système inconscient tandis que les processus secondaires correspondent davantage au système préconscient-conscient. Dans les processus primaires, l'énergie psychique s'écoule librement. Elle est dite « libre » et investit les représentations selon le modèle de la satisfaction hallucinatoire du désir, le rêve étant le prototype de ce type de ce fonctionnement dans lequel opèrent des mécanismes d'une grande labilité comme le déplacement et la condensation. Le modèle de l'identité de perception prévaut alors, le psychisme cherchant à reproduire de façon hallucinatoire les expériences de plaisir antérieures. En revanche, dans les processus secondaires, l'énergie psychique doit être « liée » pour que les représentations soient investies de façon stable, transformant ainsi le mode de satisfaction qui se trouve "secondarisé ». L'identité de pensée domine alors - la source de plaisir n'est plus la reproduction à l'identique d'une source de plaisir précédente - en régulant et en inhibant les processus primaires et leurs modes de satisfaction. Le psychisme doit ainsi, dans sa relation au monde, sacrifier une partie de sa liberté dans son rapport au plaisir, pour être suffisamment en phase avec celui-ci, et le «flux associatif » s'en trouve d'autant diminué.

L'associativité lors des séances aura pour but de relancer l'activité associative intrapsychique du patient. Ce mécanisme n'est pleinement efficace que si celle-ci est doublée d'une deuxième forme d'associativité, l'associativité intersubjective, une « co-associativité » comme la nomme René Roussillon [55], qui implique d'associer librement en présence d'un Autre, de s'adresser à soi-même par une adresse, directe ou indirecte, à autrui. L'altérité est donc fondamentale dans le processus associatif : il ne s'agit pas d'exprimer librement les secrets de sa vie psychique la plus intime de manière solipsiste; l'activité associative doit trouver les conditions lui permettant de se déployer dans la relation intersubjective. Comment opère alors cette coassociativité sur le plan thérapeutique ?

C'est tout d'abord, à un niveau très « primaire ", l'échange avec autrui et le système de «copensée » [68], la «co-psychéité » [32] relative à la situation thérapeutique, qui, lorsqu'une alliance thérapeutique suffisante est installée, favorise un travail de contenance permettant de « décontaminer», par la dynamique transférentielle, ce qui demeure en souffrance chez le patient. Ofra Eschel [15] décrit ainsi un «processus d'être ensemble» (togetherness) qui constitue une associativité de la présence à autrui particulièrement liée aux affects. Cette associativité est le terreau nécessaire pour que puissent émerger des «moments de rencontre » [64] durant lesquels aussi bien le clinicien que le patient ont le sentiment que se joue, ne serait-ce que l'espace d'un instant, une étape clef du travail thérapeutique, fruit, le plus souvent, d'une longue élaboration antérieure.

Il est possible de distinguer cette associativité primaire d'une associativité davantage secondarisée qui se trouve liée à la «prise de conscience» au sens large, c'est-à-dire à la manière dont le sujet se réapproprie une modalité du rapport à lui-même par le biais de l'activité 
réflexive. Ce processus dévoile une partie du réel énigmatique par une intelligibilité, une logique du sens, qui condense et développe les potentialités signifiantes. Cette prise de conscience réflexive peut advenir dans bien des contextes - elle n'est pas réductible au champ de la psychothérapie - mais le dispositif thérapeutique vise à condenser dans l'espace et le temps la potentialité de tels dévoilements. Ce passage par la réflexivité permet au sujet de «resentir » ou de «re-connaître » une expérience restée en souffrance [54], de sorte que la réflexivité de soi à soi ne peut advenir que par le biais d'une réflexivité de soi à l'autre. Réflexivité et associativité partagent ainsi la nécessité de se déployer par le biais de l'extériorité : ce qui ne peut se représenter et se symboliser par le biais de la réflexivité intrapsychique demeure un reste en souffrance en attente d'un Autre.

Certaines expériences non métabolisées viendront ainsi «boursoufler » [54]l'expérience actuelle dans les champs perceptif et transférentiel dans l'espoir que celle-ci puisse être partagée et reconnue [56]. Le patient «agit » alors à son insu - l'agieren freudien -, dans le cadre des séances, ce qui demeure en souffrance de ses relations antérieures. Cette logique peut, par exemple, donner lieu, dans certains cas extrêmes, aux craintes de l'effondrement telles que décrites par Donald Winnicott [70], conséquences d'agonies primitives précoces qui infiltrent progressivement la vie psychique du patient au fil des séances. Le même type de logique pourra occasionner dans certains cas un retour en entretien, sous forme hallucinatoire, de vécus traumatiques [4]. Le travail d'intégration, de métabolisation et de transformation qui opère alors par le biais de cette associativité qui se veut «débridée », nécessite que l'aspect énigmatique du psychisme s'exprime, notamment dans le transfert, «fragment par fragment », «pièce par pièce », comme le propose Freud [26]. Le psychisme ne semble, en effet, pouvoir se réapproprier une «indigestion psychique » que de façon secondarisée, par «petits bouts », au risque de déclencher, dans le cas contraire, une embolie des processus de symbolisation. Un «transfert » et une polymorphie de l'associativité permettra alors qu'un travail de traduction s'effectue auprès du patient, lointain écho des processus de transformation opérés par la mère auprès de son enfant, bien décrits, notamment, par Wilfred Bion avec la «fonction alpha » [2].

\section{Modèles de l'associativité en psychologie cognitive et dans les neurosciences}

La psychologie cognitive et les neurosciences ont développé des modèles qui étayent et enrichissent les conceptions de l'associativité que nous venons d'évoquer brièvement, dans un contexte de rapprochement entre théories psychanalytiques et travaux des neurosciences cognitives [33], [40], [46], [47], [61]. Notre objectif sera donc de «croiser les regards » [32] vers de nouvelles perspectives théoriques et cliniques concernant le champ de l'associativité selon une logique de «compatibilité » des modes de représentation de l'activité psychique.

Le fonctionnement associationniste du psychisme, supposé par Freud dès L'Esquisse d'une psychologie scientifique, en 1895 - et présent dans toute une partie des publications de son époque - se retrouve dans les théories computationnelles actuelles, dites « connexionnistes ». Celles-ci se distinguent des modèles antérieurs relevant davantage de modes «symboliques », à l'instar du fonctionnement d'un ordinateur, dans lesquels le psychisme est représenté comme un opérateur manipulant une suite de symboles [66]. Selon la perspective connexionniste, la construction du psychisme s'effectue par empreintes successives de l'environnement sur des sous-assemblées de neurones. Il existerait ainsi une «matière psychique première » [20], brute et parcellaire, retravaillée en permanence par des phénomènes de «ré-écriture » (rewriting) [1] provenant de la «construction » et de la « déconstruction » des connexions entre cellules nerveuses. Plus précisément, selon la théorie du Darwinisme neuronal de Gerlad Edelman [14], ces empreintes successives conduisent à la sélection et au renforcement de groupes neuronaux spécifiques. Ceux-ci donnent naissance à des «cartes neuronales » 
interconnectées selon des processus de «résonance» mis en évidence par des études par électroencéphalogramme [38], dans lesquelles ces groupes neuronaux se synchronisent à des fréquences caractéristiques ${ }^{1}$. Cette hétérogénéité sur le plan biologique interroge son pendant sur le plan de l'hétérogénéité psychique et de l'associativité qui lui est liée.

L'hétérogénéité des modes de fonctionnement psychiques est également l'objet des travaux de Daniel Kahneman, Professeur de psychologie à l'université de Princeton et prix Nobel d'Économie pour ses recherches portant sur la théorie des perspectives. Kahneman s'est intéressé de près à la compréhension et à la modélisation des biais de raisonnement [36] qu'il a étudiés par de petites expériences ingénieuses ${ }^{2}$. Il décrit ainsi un premier mode de fonctionnement psychique nommé « Système 1 » [16], [37]. Rapide et imprécis, il est lié à un fonctionnement intuitif, à des sentiments et des inclinaisons automatiques, quasi instinctives, façonnées par l'expérience. Le système 1 construit des causalités logiques hors de la sphère de la conscience, étant par ailleurs facilement influencé par les phénomènes de suggestion et d'amorçage ${ }^{3}$. L'humeur et l'engagement cognitif sont également des éléments ayant un impact majeur sur son fonctionnement. Le système 1 est aussi sensible aux effets de « halo » et produit un ensemble d'approximations dans le raisonnement que Kahneman a étudiées avec soin avant de les appliquer au champ de l'économie comportementale. Il découle de ces travaux que le cerveau humain semble naturellement partisan du moindre effort et préfère s'en tenir aux informations les plus «accessibles » selon des logiques relevant de «l'heuristique ». Lorsque les relations ainsi produites ne sont pas «secondarisées », c'est-à-dire «validées » par le système 2, les sujets tendent à faire davantage d'erreurs cognitives. Kahneman décrit ainsi une «machine associative » et des logiques de «cohérence associative » qui caractérisent le système 1, afin de permettre au psychisme de spontanément et automatiquement construire et produire du sens à partir de liens de causalité sous-jacents.

Kahneman rejoint ainsi, par une méthodologie et une perspective originales, la distinction que proposait Freud entre processus primaires et processus secondaires qui, comme nous l'avons évoqué plus tôt, constitue une première «cartographie» de l'hétérogénéité et de l'associativité psychique. Il est d'ailleurs possible de mettre en parallèle ces deux niveaux dans le tableau ci-dessous qui combine les approches de Freud [57] et Kahneman [36] :

$\begin{array}{ll}\text { Processus primaires }- \text { Système } 1 & \text { Processus secondaires - Système } 2 \\ \text { Temporalité courte } & \text { Temporalité lente } \\ \text { Automatique } & \text { Réfléchi } \\ \text { Inconscient } & \text { Conscient } \\ \text { Pas de négation } & \text { Négation } \\ \text { Intuitif } & \text { Rationnel } \\ \text { Perceptif } & \text { Conceptuel } \\ \text { Principe de plaisir } & \text { Principe de réalité }\end{array}$

\footnotetext{
${ }^{1}$ Ce qui ouvre d'ailleurs le champ relativement peu exploré des états modifiés de conscience [7] dans leur relation aux modes d'associativité. Cette question était d'ailleurs centrale dans Les études sur l'hystérie[29], Freud et Breuer repérant le lien réciproque entre état hypnoïde et traumatisme, qui a été depuis étudié en particulier dans le cadre des recherches sur les Fantasy Prone Personality [31], [69].

${ }^{2}$ Par exemple : «Une batte de baseball et une balle coûtent 1,10 dollars. La batte coûte un dollar de plus que la balle. Combien coûte la balle ? » Même au sein de populations d'étudiants provenant d'universités américaines prestigieuses, près d'un étudiant sur deux donne une réponse erronée (la bonne réponse est 0,05 centimes).

${ }^{3}$ Certaines expériences menées par Kahneman confirment l'influence de détails subtils sur les processus de pensée (p. 74), en particulier au niveau du système 1 et de l'associativité. Par exemple, selon le type d'image placé devant une boite servant à recueillir des dons, l'argent perçu pourra varier considérablement. Ces expériences étayent de manière objective l'idée que le cadre « symbolise » l'associativité de manière automatique et inconsciente.
} 
Processus primaires - Système 1

Énergie libre
Processus secondaires - Système 2

Énergie liée

${ }^{a}$ La distinction selon le niveau d'entropie sera explicitée dans la suite de l'article.

Les deux modèles ne se recoupent pas entièrement, d'autant que le modèle freudien évoluera au fil du temps. Néanmoins, les points d'achoppement nous paraissent suffisamment nombreux pour qu'il y ait une certaine pertinence à un tel rapprochement, à condition d'en souligner également les écarts. Ainsi, si Kahneman analyse les modes de fonctionnement psychiques essentiellement sur le plan des mécanismes cognitifs et d'une forme d'hétérogénéité du raisonnement, les conceptions freudiennes représentent une théorie de l'économie psychique du sujet qui traite de sa construction psychoaffective. En somme, Freud traite davantage de la question du « pourquoi » tandis que Kahneman s'attache plutôt au « comment ». Leurs théories se rejoignent néanmoins à leurs frontières concernant la bi-polarité fondamentale des processus psychiques et leurs différentes formes d'associativités.

Cette dichotomie du fonctionnement psychique est étudiée plus précisément par Freud du point de vue de la « liaison » psychique, c'est-à-dire selon la manière dont le psychisme tente de contenir et de «lier » l'énergie nerveuse et les excitations externes. Freud et Breuer ont été en ce sens influencés par les physiciens de leur époque, en particulier par les théories d'Hermann von Helmholtz à partir desquelles Breuer proposa la distinction entre «énergie tonique » et «énergie cinétique» avant que Freud ne développe l'opposition énergie libre/énergie liée qui différencie les modes de fonctionnement primaires et secondaires [39].

Le concept d'«énergie libre » est également très présent dans les travaux du neuroscientifique Karl Friston [30]. Celui-ci offre un éclairage intéressant sur la thématique de l'associativité en interrogeant la pertinence actuelle du modèle freudien tout en étudiant les contraintes neuronales de l'associativité du point de vue neurobiologique et computationnel. Le modèle de Friston est relativement complexe et nous allons à présent tenter de le synthétiser. Il est tout d'abord nécessaire d'avoir à l'esprit que tout organisme vivant doit résister à la deuxième loi de la thermodynamique, à savoir la tendance spontanée de tout système à tendre vers un état de désorganisation. Le niveau d'organisation d'un système est habituellement évalué selon son degré d'entropie : plus celle-ci est élevée, plus le niveau de désorganisation est important. Les organismes biologiques doivent donc lutter de manière primordiale contre un degré d'entropie élevé qui pourrait signifier leur mort. Cette entropie élevée peut tout d'abord provenir d'une source externe, c'est-à-dire la désorganisation produite par l'environnement qui se transfère dans l'être vivant par le biais de l'excitation de ses organes sensoriels. Mais une augmentation de l'entropie peut également provenir de l'organisme luimême, issue notamment de la tendance naturelle et spontanée de la «matière douée de vie » [27] à se désorganiser ${ }^{4}$.

Le psychisme est une sous-catégorie du vivant qui obéit aux mêmes logiques. Il est conçu par Friston comme un «modèle de travail » (working model) dont la fonction essentielle est de limiter les états de désorganisation. Pour cela, le cerveau produit une représentation cohérente et prédictive de l'environnement extérieur, dans la continuité des hypothèses d'Helmholtz du

\footnotetext{
${ }^{4}$ Ce qui rejoint les intuitions freudiennes concernant la nature de la pulsion de mort.
} 
cerveau en tant que " machine inférentielle », tendant à déduire les causes probables des stimuli sensoriels. Mais ce travail de prédiction n'est pas parfait et il en résulte toujours un écart entre les représentations du psychisme (sous-tendues par le réseau neuronal) et les données perceptives du monde environnant. Friston nomme cet écart énergie libre et Freud en faisait un usage très proche. Le psychisme ne peut en effet simuler l'ensemble des situations de rencontre avec l'environnement et il se produit donc parfois des états de «surprise ». Pour limiter l'augmentation de la désorganisation interne, c'est-à-dire l'augmentation de l'énergie libre et des effets de surprise, le cerveau développe un modèle probabiliste bayésien ${ }^{5}$ de la cause potentielle de ses sensations en fonction de ses croyances antérieures, permettant ainsi de réduire a priori l'énergie libre.

Ce modèle se construit, selon Friston, à partir des sensations, des actions et des interactions du sujet avec son environnement en vue d'évaluer la pertinence de ce modèle. Plus celui-ci est fiable - ce qui restreint l'écart entre monde interne et monde externe -, et plus l'effet de "surprise » induit par la rencontre avec l'environnement diminue, de même que l'entropie générée par cette rencontre. Les différents «états» occupés par le système biologique nécessaires pour être en phase avec son environnement sont alors réduits. Cette relation sera également modulée par les actions de l'organisme biologique car celles-ci lui permettent de modifier son rapport à l'environnement et donc les effets d'entropie auxquels il pourrait être confronté. Ces processus seraient plus précisément organisés selon Friston en fonction d'un «modèle hiérarchique », variation empirique des modèles bayésiens, dans lequel les niveaux plus élevés du fonctionnement cérébral exercent une contrainte sur les niveaux inférieurs : «la suppression de l'énergie libre signifie que chaque niveau tente d'expliquer les erreurs de prédiction à son niveau et au niveau situé en dessous $»^{6}[30]$ (p. 295). Il existerait ainsi des relations complexes entre les types de neurones, leur niveau hiérarchique et les processus topdown et bottom-up qui modifient les processus de neuromodulation et les mécanismes de plasticité associative à des niveaux plus élevés. Cette théorie a par ailleurs pour avantage d'être modélisée sur le plan computationnel et précise ainsi les contraintes neuronales de l'associativité.

\section{Neuropsychanalyse, mode par défaut et associativité}

Dans un récent articule intitulé The Conscious Id [60], Marks Solms, l'un des fers de lance de la recherche en neuropsychanalyse [61], tente d'articuler un certain nombre de travaux en neurosciences contemporains, en particulier les travaux de Karl Friston que nous venons d'évoquer, avec les modèles psychanalytiques et les logiques de l'associativité. Solms critique tout d'abord une vision cortico-centrique du psychisme, présente dans l'œuvre freudienne de même que chez la plupart de ses contemporains, voire dans certains travaux de neurosciences actuels, qui considèrent le cortex comme étant le centre de la conscience. Solms suppose, en réalité, que la conscience serait avant tout une conscience affective liée au système réticulaire ${ }^{7}$. Une forme de conscience serait donc présente d'emblée ${ }^{8}$ et les états de conscience représenteraient essentiellement des états affectifs, comme le soulignent également les recherches d'Antonio Damasio [10] et de Jaak Panksepp [44]. En conséquence, et d'un point de vue conceptuel, la conscience dépendrait en premier lieu de logiques appartenant à des processus décrits par Freud comme relevant du Ça et non du Moi. Quant au cortex, sa fonction

\footnotetext{
${ }^{5}$ Créées par Thomas Bayes, les statistiques bayésiennes consistent à déduire une probabilité en fonction d'évènements qui se sont déjà produits. Ce modèle est de plus en plus utilisé dans le champ de la psychologie empirique [12].

${ }^{6}$ Traduction de l'auteur.

${ }^{7}$ Structure neurologique du tronc cérébral, influencée par les stimuli somatiques et émotionnels, notamment responsable du tonus musculaire et de l'état de vigilance.

${ }^{8}$ Solms s'appuie sur un ensemble de données issues des neurosciences, notamment le cas d'enfants dont le développement du cortex a été très fortement altéré dès la naissance, les enfants souffrant d'hydrocéphalie, [60] (p. 11) et manifestant pour autant tous les signes d'une forme de conscience primaire.
} 
essentielle serait non pas de produire la conscience mais plutôt de contribuer à « stabiliser » les objets de la perception. Le cortex permettrait plus précisément l'émergence d'un « espace de mémoire représentationnel » au sein duquel pourrait se déployer l'associativité du sujet. Les représentations seraient essentiellement de nature inconsciente et ne deviendraient conscientes que lorsqu'elles seraient l'objet de l'attention, étant alors transformées par le cortex en un matériau suffisamment stable pour être objet de la mémoire de travail, comme le souligne Solms : "La fonction essentielle du cortex est de générer des représentations stables de "solides mentaux" qui, lorsqu'ils sont activés ou investis par la conscience affective, permettent au moi de se représenter lui-même dans le monde et de penser $\gg^{6}[60]$ (p. 14). Cette conception intéresse d'ailleurs de façon plus générale la thématique de la figurabilité psychique telle qu'elle a été développée, notamment, par César et Sara Botella [5], en tant que «travail de transformation qui tend à une mise en figurabilité comme le meilleur moyen d'effectuer une mise en intelligibilité de l'hétérogénéité psychique » [3] (p. 5). Se pose ainsi la question du passage d'un niveau de fonctionnement psychique à un autre et son impact dans la dynamique psychique. Quelles sont, plus précisément, les conséquences de ce modèle sur les théories de l'associativité ?

Les processus primaires appartiendraient à une première forme de conscience primitive, caractérisée par les affects, qui précède une conscience secondarisée ayant pour fonction essentielle la stabilisation des objets mentaux. La pluralité des modes de fonctionnement psychique et la polymorphie de l'associativité seraient la face émergée de cette dichotomie du fonctionnement cérébral. Cette hétérogénéité conduirait ainsi à des particularités et des logiques associatives influencées par le passage des processus primaires aux processus secondaires en fonction de la manière dont l'énergie libre va se trouver « liée » par les processus secondaires. C'est par le biais de l'activité consciente que l'énergie libre sera liée, permettant la stabilisation et la figuration consciente des représentations mentales tandis que l'énergie libre, non liée de par sa nature même, sera de ce point de vue de l'affect non transformé, et donc, non représenté 9 . Comment opère alors ce passage de la conscience primaire affective à la conscience secondarisée, de l'énergie libre à l'énergie liée, et quel impact cela a-t-il sur les logiques associatives ?

Selon Friston et Carhart-Harris [8], ce passage se ferait en particulier par le biais du "mode par défaut» («default mode network ») (DMN) [50]. Sans rentrer dans un «plaquage» théorique qui viserait des corrélations simplistes et hasardeuses entre entités conceptuelles psychanalytiques et neurobiologiques, le DMN a des propriétés qui méritent d'être soulignées. Il correspond tout d'abord à un réseau qui se développe durant l'enfance et qui conduit à l'interconnexion de plusieurs zones anatomiques, en particulier le lobe temporal médial, le cortex préfrontal médial, le cortex cingulaire postérieur, le précunéus et d'autres régions avoisinantes du cortex pariétal [6] qui ont pour particularité d'être actives à l'état de repos. On notera ainsi que la variation de l'activité cérébrale globale est seulement de $5 \%$ entre la focalisation du cerveau sur une tâche et son état de repos [50], soulignant ainsi le travail psychique qui opère indépendamment de la relation à l'environnement. Le DMN consomme plus d'énergie que n'importe quelle autre zone du cerveau et semble faire office de "chef d'orchestre » de ses fonctions globales. Cette forte activité cérébrale est probablement liée à la haute densité associative entre les régions qui le composent. L'activation du DMN a également pour particularité de diminuer l'activité des niveaux d'organisation inférieurs et semble opérer de ce point de vue un travail de modulation des excitations internes et externes. Il est en effet très éloigné des niveaux purement sensoriels et se trouve engagé dans des opérations plus élevées relevant de la méta-cognition et de la réflexivité, comme le montrent plusieurs protocoles d'imagerie [8]. Des oscillations spontanées synchrones dans le cortex cingulaire

${ }^{9}$ Cette perspective amène plus largement à la vaste question du « binding » et des processus d'inhibition étudiés par les psychanalystes du côté de la négativité. Les logiques de l'associativité sont en effet dessinées en creux par celles de la négativité. 
postérieur, en particulier dans l'alpha de $8-13 \mathrm{~Hz}$, sont par ailleurs un marqueur neurologique de son fonctionnement, ce que Carhart-Harris et Friston relient à un éventuel travail d'intégration du Moi [8] (p. 2). On notera enfin que l'activation du DMN est inversement proportionnelle au système attentionnel ${ }^{10}$ et que son activité diminue avec l'âge ainsi que chez les personnes souffrant de troubles de l'attention.

À partir de ces différents éléments, Carhart-Harris et Friston émettent l'hypothèse que le DMN serait un équivalent neurobiologique du «Moi », en tant qu'entité dont la fonction essentielle serait la gestion et l'articulation des excitations internes et externes. Plus précisément, selon Friston [30], l'activité consciente, liée aux processus de traitement du DMN, serait une mesure temporaire du travail d'adaptation du psychisme à la réalité. Il s'agirait alors pour le psychisme d'une tentative de « traiter » tout écart entre réalité interne et réalité externe. Ainsi, le but fondamental du Moi serait de limiter son activité en recherchant un mode «automatique » qui permettrait que les ajustements nécessaires entre réalité interne et réalité externe soient minimisés. Selon Solms [60], ce travail d'articulation et de prédiction qui obéit au principe de constance de la réalité psychique opère lors du passage des processus primaires aux processus secondaires. Ainsi, par exemple, le but de l'apprentissage conscient serait avant tout l'automatisation et l'adéquation des mécanismes de pensée à l'environnement, le monde devenant d'autant plus «prédictible » à mesure que les effets de surprise diminuent. Dans la continuité des hypothèses de Friston, Solms propose plus précisément que la conscience aurait pour fonction principale d'effectuer ce «travail de prédiction» par le biais des «affects de conscience » (affects of consciousness), ceux-ci «caractérisant », " labélisant » la qualité de l'expérience vécue et auto-informant le sujet de son adéquation à son environnement. Le rapport premier au monde serait donc un rapport affectif lié de manière intrinsèque au principe de plaisir-déplaisir. La conscience aurait ainsi pour fonction essentielle de « retravailler » les affects en souffrance provenant d'un douloureux écart entre monde interne et monde externe. Lorsque les effets de surprise diminuent, la conscience n'est alors plus nécessaire, de la même manière que le danseur ne réfléchit pas au geste reproduit mille fois.

L'appareil de langage et les mots permettront de réguler secondairement ces logiques affectives premières, ouvrant la voie à des formes d'associativité obéissant à d'autres principes. Les signifiants participeront ainsi des processus secondaires en rajoutant un «délai » supplémentaire donnant une coloration particulière à l'activité consciente. Il se produit alors un « transfert » des logiques associatives et émotionnelles primaires que nous venons d'évoquer, dans l'appareil de langage, un transfert de l'affect dans les mots, ceux-ci gardant la trace de ce passage par le corps sous forme d'une « corporéité » et d'une « matérialité » particulières [58].

\section{Modèles de l'associativité et cerveau entropique}

Comme nous venons de l'évoquer, le cerveau aurait évolué afin de simuler précisément son environnement de manière à ce que les effets de surprise soient diminués. L'associativité peut être considérée de ce point de vue comme l'écho de ce processus, c'est-à-dire l'intériorisation de ce mode de construction de la réalité psychique en lien avec la réalité externe. L'associativité intrapsychique apparaît ainsi comme le fruit d'une associativité externalisée ; elle est le reflet, la trace, de l'associativité de l'environnement auquel a été confronté le sujet.

Carhart-Harris prolonge et étend ces réflexions par l'hypothèse du «cerveau entropique » [9] qui aide à penser plus finement ces évolutions conceptuelles provenant du champ des neurosciences et les modèles de l'associativité. Pour cela, il s'appuie sur le concept d'entropie, qui rappelons-le, désigne le degré d'organisation d'un système. De ce point de vue,

\footnotetext{
${ }^{10}$ Le système attentionnel n'est en réalité pas un système unifié. On peut distinguer un salient system et un dorsal attention system. Il existe une relation proportionnelle entre eux. Le système hiérarchique est constitué au niveau le plus élevé du DMN et les deux systèmes attentionnels se situent à des niveaux intermédiaires.
} 
les structures hiérarchisées auxquelles nous avons fait allusion précédemment se situent au sein d'un continuum, sur le plan entropique, selon leur niveau d'organisation. La conscience primaire, décrite par Solms, correspond ainsi à une entropie élevée; elle est moins «méticuleuse » dans son rapport au monde, elle est plus «perméable » au réel - comme le montrent également les recherches de Kahneman - et se trouve davantage influencée par des facteurs internes et externes. La conscience secondarisée, en revanche, se caractérise par sa capacité à diminuer l'entropie, issue de la conscience primaire, en organisant et en contraignant la cognition. Ainsi, les états plus primaires sont davantage «entropiques » que les états secondarisés et les processus sont plus flexibles dans la conscience primaire que dans les processus secondarisés. La conscience «normale », telle qu'on la conçoit habituellement dans le sens commun, est donc un état de haute organisation correspondant à une faible entropie.

Quand le rapport à l'environnement se complexifie et devient source d'une grande incertitude, le sujet est en quelque sorte "imprégné » par cette incertitude qu'il doit juguler. Il pourra alors réagir de différentes manières. Par exemple, la «pensée magique » sera une manière d'interpréter le monde en fonction de ses désirs lorsqu'un niveau d'entropie élevé « déborde » les processus secondarisés. Dans les états dépressifs, le psychisme serait également en difficulté pour juguler l'incertitude issue de niveaux plus primaires du fonctionnement psychique $^{11}$. On observe alors, par imagerie, une hyperactivation du DMN, conséquence d'une introspection hypertrophiée provenant d'une tentative désespérée du Moi de juguler les processus primaires. Carhart-Harris décrit plus finement ces logiques à partir de la «théorie de l'auto-organisation critique » (self-organized criticality). Celle-ci porte sur la manière dont un système complexe, déséquilibré par une entrée d'énergie nouvelle, développe des propriétés spécifiques lorsqu'il atteint un point critique. Celui-ci est situé dans une étroite zone de transition entre les deux positions extrêmes de chaos et d'ordre d'un système. Trois propriétés se développent alors : des états métastables ou stables de façon transitoire, une sensibilité à la perturbation, une tendance à des processus de type «avalanche » qui pourraient avoir leur corrélat dans le fonctionnement du Moi.

Il est possible d'étudier expérimentalement ces variations d'organisation et d'associativité par le biais des psychédéliques - en particulier la psilocybine -, comme l'a tenté l'équipe de Carhart-Harris [9]. Les psychédéliques produisent en effet un état prototypique de conscience à haute entropie. Ils altèrent la conscience par une désorganisation de l'activité cérébrale qui se traduit, en particulier, par une diminution significative de l'activité des zones cérébrales clefs du DMN. Ils engendrent ainsi des états d'insight profonds concernant le «Soi », souvent rapportés comme un sentiment de dissolution du Moi et de ses frontières, du registre du sentiment océanique [28]. Les phases de sommeil paradoxal, les périodes psychotiques initiales et aiguës, certaines problématiques épileptiques sont également considérées comme des états de régression cognitive du registre de la «conscience primaire». Ainsi, comme le supposait Freud, le rêve et la psychose partagent probablement un modèle de conscience des premières formes de structuration du cerveau dominant dans l'enfance, la méta-cognition ne se développant que secondairement. On notera également que la régression à ces états archaïques à partir d'états davantage secondarisés se fait de manière discontinue par une "phase de transition » qui serait potentiellement repérable en clinique.

Une distinction est ainsi faite entre deux styles de cognition, le premier étant caractéristique de l'état de conscience de l'adulte tandis que le second, présent dans l'enfance, pourrait émerger à la faveur de mécanismes de régression. Ces états de conscience correspondent à certaines fréquences de l'activité neuronale, notamment la puissance des ondes alpha, celle-ci étant corrélée à l'activité réflexive [9]. Certains rythmes cérébraux seraient en particulier associés à une diminution de l'entropie du fait d'une augmentation des échanges d'information entre

\footnotetext{
${ }^{11}$ On pourrait d'ailleurs considérer que le clinicien fait office, de ce point de vue, de « Moi auxiliaire » venant étayer le processus de transformation en souffrance chez son patient.
} 
assemblées de neurones. La prise de psilocybine induit plus précisément une diminution de l'activité cérébrale dans le spectre alpha corrélée à un sentiment subjectif de désintégration. Sous psychédéliques, le cerveau se comporte donc de manière plus aléatoire, sa connectivité et son fonctionnement hiérarchique deviennent anarchiques et, paradoxalement, l'associativité se débride, régressant à des modes de fonctionnement primaires ${ }^{12}$. Les effets thérapeutiques des psychédéliques reposent alors sur une forme « extrême » de symbolisation que l'on retrouve en psychothérapie de manière atténuée. Le déploiement de l'associativité, et le passage par des états entropiques élevés qui lui est corrélée, permettra qu'advienne un certain "lâcher-prise » nécessaire à la psyché pour relancer les processus de symbolisation. L'associativité apparaît ainsi comme le point nodal d'évaluation du rapport aux processus d'intégration de la rencontre entre monde interne et monde externe ${ }^{13}$.

\section{Cliniques de l'associativité, processus de symbolisation et transmodalité symbolisante}

Dans quelle mesure les points théoriques évoqués précédemment entrent-ils en résonance, voire infléchissent, les modèles cliniques de l'associativité et de la symbolisation ? Notons tout d'abord que les travaux que nous venons d'évoquer s'orientent essentiellement vers une compréhension du fonctionnement cognitif «normal ». Ce seront à l'inverse, habituellement, les « ratés », les «blocages », les «failles » de la «machine associative » et la manière de la « relancer » qui intéresseront le clinicien, ce qui ajoute un degré de complexité supplémentaire. Il convient plus précisément de déterminer de quelle manière le cadre psychothérapique, en particulier le cadre d'inspiration psychanalytique, opère comme un dispositif ouvrant la voie à l'émergence de formes associatives symboligènes et «efficientes ». Ainsi, lorsqu'il est proposé ou suggéré au patient de «tout dire » ou de dire «tout ce qui lui vient à l'esprit », une associativité particulière se met progressivement en place au fil des rencontres afin de « délier » l'associativité. Celle-ci apparaît alors dans toute sa complexité : multiple et protéiforme, interne et externe, intrapsychique et intersubjective, concernant aussi bien des sensations, des émotions, que des images ou des souvenirs. Le sujet utilise également les différents «langages » en sa possession : la respiration, le placement de son corps dans l'espace, le rythme et la succession des expressions corporelles et faciales, les mots et l'ensemble des structures linguistiques qui déterminent le langage parlé, chacun de ces registres permettant l'expression de la vie psychique.

Les logiques de l'associativité dépendent alors des différents modes de symbolisation, ceuxci étant à considérer, au sens large, comme la manière dont le sujet partage son expérience interne en même temps qu'il la transforme, tout en se ressaisissant de celle-ci selon une logique réflexive et intersubjective. Lors du processus de symbolisation, une associativité particulière se met en place, dont les corrélats neurologiques sont peut-être à rechercher du côté du mode par défaut, favorisant l'émergence d'expériences restées en souffrance. Certains contenus psychiques semblent en effet «en attente» de ce traitement, et le cadre thérapeutique, sa temporalité propre, sa non-directivité, sont autant d'éléments qui convoquent et catalysent le processus de symbolisation. Le contenant spécifique signifié et symbolisé par le cadre psychothérapique opère ainsi comme un « espace de transformation » qui s'étaye sur une liberté et un lâcher-prise nécessaires.

Dans ce cadre, c'est tout d'abord ce que nous pourrions appeler une «associativité primaire », une associativité des affects de l'ordre de la conscience primaire décrite par Solms,

\footnotetext{
${ }^{12}$ Pour autant, s'il paraît cohérent et nécessaire, d'un point de vue théorique, de distinguer ces deux modes de fonctionnement et leurs éventuels corrélats neurobiologiques, il est fort probable que ceux-ci fonctionnent en parallèle. Dans les recherches de Kahneman [37], il apparaît en effet que le système 1 et le système 2 travaillent souvent de concert.

${ }^{13}$ Des formes spécifiques d'associativités particulièrement symboligènes, des «symbolisations extrêmes », pourraient en outre survenir lors de certaines expériences « exceptionnelles » [17], [48], [49].
} 
qui « dialogue » durant les séances. Cette forme de conscience partagée, cette « co-associativité affective », repose sur des processus archaïques et découle de la présence du clinicien qui sera dépendante, de ce point de vue, de ses compétences régressives aux niveaux les plus primaires de la psyché, comme le souligne notamment Catherine Parat [45] : «Il s'agit de la possibilité d'une relation interhumaine qui s'établit directement et régressivement sur un mode préverbal, anté-verbal, où l'affect de l'un entre en résonance avec l'affect de l'autre. C'est peut-être la seule voie qui permette l'abord et la mobilisation des éléments, des sédiments du refoulé primaire » (p. 171). La position du clinicien permet alors un «transfert de base », selon Catherine Parat [45], un «partage intersubjectif de l'affect» selon Daniel Stern [64], « une activité d'accompagnement » pour Christian David [11]. Ce partage d'affect est une première forme d'associativité intersubjective et indifférenciée qui permet le partage d'un "reste psychique » non symbolisé. Cette associativité primaire peut être particulièrement archaïque et "perméable », comme le montrent les travaux de Michel De M'Uzan sur la pensée paradoxale [42] ou les systèmes de co-pensée de Daniel Widlöcher [68], caractérisés par des formes de transmissions psychiques d'inconscient à inconscient [18].

Ce premier niveau d'échange peut donner lieu à une certaine inconscience, une forme de «non-représentation », voire un nécessaire «désir d'irreprésentation » [11]. Il apparaît ainsi que certains processus de symbolisation nécessitent une part d'obscurité suffisante. À trop chercher à savoir, voire à « rationaliser » ce qui se joue avec le patient, on prend ainsi le risque de limiter les processus sous-jacents qui, en passant des processus primaires à des registres secondarisés et conscients, perdent dans ce détour par la réflexivité consciente leur part symboligène, donnant lieu alors à de pseudo-progrès thérapeutiques [62]. Cette première forme d'associativité, qui entretient des rapports complexes avec les processus de mémoire et de perlaboration, se devra donc de garder une part d'indicible. Les processus psychothérapiques que l'on retrouve, par exemple, dans le champ de l'hypnose, avec la pratique d'un Milton Erickson [35] ou d'un François Roustang avec le concept de «perceptude ${ }^{14}$ [59], sont une illustration des rapports particuliers qu'entretiennent les processus de symbolisation avec l'activité consciente. L'hypnothérapeute cherchera en effet, le plus souvent de manière métaphorique, par la confusion ou le détournement de l'attention, à relancer les processus de symbolisation en dehors de la sphère consciente, ou du moins, hors d'une compréhension raisonnée et rationnelle des logiques sous-jacentes à ceux-ci [31], [69].

À un niveau de structuration psychique plus élaboré, le passage par les mots et la chaîne signifiante conduit à « briser » l'associativité primaire et le « halo représentatif » [45] solipsiste et hallucinatoire qui caractérise les formes associatives primaires. Émerge alors une « associativité secondarisée » qui nécessite que le sujet sorte de l'état de régression dans lequel il se situe pour intégrer, à des niveaux plus élevés, l'expérience en cours d'élaboration. Le travail de transformation opère alors essentiellement par le biais de l'activité consciente - probablement en étayage sur le «mode par défaut»-, et rejoint, en ce sens, la fonction du « modèle de travail » décrit par Karl Friston visant à réduire l'entropie. Dans ce cas de figure, l'activité consciente secondarisée devient prépondérante dans le processus de transformation psychique. Le cadre psychothérapique pourrait être considéré, dans cette perspective, comme un espace aux propriétés particulières, dont les caractéristiques, symbolisées notamment par le cadre, permettent de retraiter les expériences difficilement métabolisables. Celles-ci sont alors réélaborées sur les plans corporel et émotionnel par le biais d'une conscience affective autoinformant le sujet quant à l'adéquation de son comportement à son environnement.

Le mécanisme décrit par les neurosciences, aux niveaux primaire et secondaire, serait donc le processus «normal» par lequel le sujet entretient un rapport harmonieux à son

\footnotetext{
${ }^{14}$ François Roustang définit la perceptude comme « un état de veille intense, à l'instar du sommeil profond à partir duquel nous rêvons. De même que ce sommeil profond conditionne l'éclosion du pouvoir de rêver, de même cette veille intense nous fait accéder au pouvoir de configurer le monde ».
} 
environnement mais, dans certaines conditions, celui-ci se « coupe » de l'inscription subjective de l'expérience vécue. Le sujet garde alors en lui la trace de la transformation partielle de cette expérience inachevée et le cadre thérapeutique relance le travail de symbolisation. Ce travail reprend là où il s'est arrêté, au niveau de la conscience affective et des processus d'intégration sur le plan émotionnel. Une expérience ne peut, en effet, être intégrée que si elle a été source suffisante de plaisir [57]. Si tel n'est pas le cas, elle tendra à se répéter sur le mode de la compulsion de répétition. Ainsi, lorsque ce processus est relancé, une partie de l'associativité se fera «à l'insu » du sujet du fait de la polymorphie de l'associativité, en deçà du travail de « stabilisation » des objets mentaux décrit par Solms, à des niveaux dépendant de la conscience affective.

Cela interroge de manière plus globale l'articulation des associativités primaires et secondaires. En séance, le sujet passe d'une idée à une autre et « déroule » consciemment une chaîne signifiante composée d'affects et de représentations [65]. Celle-ci, associée notamment à la négativité du langage et à son expression non-verbale, vectorise, de façon discrète, le passage des processus primaires aux processus secondaires, par l'intermédiaire de l'activité consciente et attentionnelle. La «rêverie » en séance décrite par Wilfred Bion, aussi bien du côté du clinicien que du patient, favorise alors l'émergence spontanée des pensées, l'accès aux logiques inconscientes et « fluidifie » l'activité psychique en même temps qu'elle la représente. Quand ce processus d'articulation se complexifie - ce qu'André Green nomme les processus tertiaires [34] - et touche des niveaux psychiques plus élaborés, secondarisés, le processus de symbolisation implique des mécanismes de stabilisation des objets mentaux qui « figent »en même temps qu'ils représentent l'activité de pensée. Ce travail de «stabilisation », découlant de l'activité du cortex selon Solms, participe du travail de transformation psychique et donne une «coloration » particulière à l'expérience consciente. En témoigne, par exemple, le vécu du patient qui devient soudainement en mesure de lier des pans de son histoire demeurés jusqu'ici inintelligibles. Il produit ainsi une « configuration signifiante » originale, une « construction » pour reprendre l'expression freudienne [19], qui organise le vécu expérientiel comme conjonction des vécus primaires et de leur reprise dans l'appareil de langage. Il se produit alors, peut-être, à ces différents niveaux, des processus de « reconsolidation » tels que décrits par Pierre Magistretti [1], ces vécus particuliers étant le signe que la trace mnésique redevient « labile ». Elle peut alors être retraitée psychiquement à différents niveaux selon les processus associatifs, aussi bien à des niveaux plus primaires que secondarisés, selon les logiques hiérarchiques décrites par Friston, les processus langagiers « régulant » les processus primaires. Les mots, le verbe, viennent ainsi au secours du corps et de l'affect restés en souffrance.

C'est plus précisément dans le passage d'une associativité à une autre forme d'associativité, et des modes de symbolisation qui l'accompagnent, que les progrès thérapeutiques se font habituellement les plus prégnants. Nous proposons de condenser ce double processus associatif et symbolisant avec la notion de «transmodalité symbolisante » qui englobe en même temps qu'elle étend les conceptions abordées jusqu'à présent. Celle-ci peut être définie comme étant la manière dont l'activité psychothérapique permet le transfert associatif entre modalités symbolisantes d'un processus psychique resté inachevé. Elle découle d'un «transfert intersubjectif d'associativité », dans un aller-retour entre clinicien et patient, ouvrant la voie à une transmodalité intrapsychique d'une forme de modalité associativité à une autre. Ainsi, la transmodalité symbolisante opère un travail de transformation de ce que le sujet ne sent pas de lui-même et qu'il tente d'explorer par le biais d'une autre modalité sensorielle. Le passage transmodal - que l'on observe au niveau prototypique et externalisé dans la relation mèrebébé [63] - favorise alors la relance du processus de symbolisation. Il s'agit d'un travail de métaphorisation de l'expérience interne qui opère selon un gradient subtil allant des formes les plus primaires et inconscientes aux processus davantage secondarisés et conscients, en étayage sur l'aspect protéiforme de l'associativité. 
L'émergence et la qualité de la transmodalité symbolisante seront repérables par les effets de « surprise », en tant qu' indice du travail de transformation provenant du passage entre modalités associatives. D'où ce paradoxe étonnant, comme le relevait déjà Théodor Reik [51], de la nécessité que le clinicien préserve sa capacité à être « surpris » ${ }^{15}$, ce positionnement permettant, en écho, de favoriser les effets de surprise chez le patient. Rappelons à ce propos que dans le modèle de Friston les effets de surprise seront redoutés par le psychisme car signifiant un écart important entre mondes interne et externe, source potentielle d'inadéquation du modèle de travail et donc risque possible d'un déséquilibre de la constance interne. Dans cette perspective, il semble que le mécanisme psychique de «surprise », qui normalement auto-informe le sujet, notamment sur le plan affectif, ne soit plus efficace - du fait de processus de sidération psychiques ? - et nécessite, pour être relancé, de passer par le clinicien et l'intersubjectivité sécure de la thérapie. La «prise de conscience » et l' " eurêka » seront les formes que pourront prendre ces effets de surprise associés à l'expérience de plaisir, menant à une amélioration soudaine du modèle opérant interne : il s'agit d'une capacité de liaison originale dont la créativité en est l'exemple paradigmatique. Que l'on songe, par exemple, dans le champ scientifique, à Newton découvrant les lois de la gravitation sous un pommier ou encore à Einstein formulant la relativité restreinte après l'observation répétée des horloges et des trains en gare de Berne. Il s'agit d'organiser de manière originale un ensemble de représentations internes, par le biais d'une médiation externalisée, support l'élaboration interne et signifiante. Ce modèle de la créativité se rapproche de celui que l'on retrouve en séance sur le plan psychoaffectif : ce qui importe, c'est la rencontre avec un objet ou un Autre dont les propriétés favorisent la relance du processus de symbolisation par l'extériorité, produisant alors une expérience subjective originale. L'expérience initiale se transfère ainsi dans le médium et permet au sujet de se ressaisir de lui-même en s'enrichissant de l'associativité transmodale de l'objet. La clinique de l'enfant offre d'ailleurs une illustration originale de ce processus, la transmodalité symbolisante s'exprimant alors essentiellement par le jeu. En effet, le travail de «stabilisation » des objets mentaux n'est probablement pas suffisamment opérant et l'enfant a besoin de passer par l'objet à condition que celui-ci «colle» suffisamment à ses prémisses d'objets mentaux comme le supposait Winnicott avec l'«espace transitionnel » et les mécanismes du «trouvé-créé » [71]. Ce principe participe probablement de la complexité de la clinique auprès des enfants, tant il est frappant de voir comment la problématique qui les occupe s'exprime de façon condensée, ramassée, selon une structure ribozimatique, lors de la première consultation. Le premier jeu de l'enfant, en particulier, ne devient souvent intelligible qu'au fil des séances et condense ce qui le fait souffrir, l'associativité lui donnant naissance étant comme « condensée » dans la médiation.

\section{Conclusion}

Les modèles de l'associativité issus de la psychanalyse et des neurosciences ont des origines communes qui participent de leur compatibilité actuelle. Le «double regard » qui découle de leur rencontre permet de rendre davantage intelligible les processus et les logiques cliniques qui œuvrent habituellement dans l'ombre. Il apparaît tout d'abord que l'activité de pensée correspond à une hétérogénéité psychique et biologique, entre processus primaires et secondaires, système 1 et système 2 . Cette hétérogénéité conduit à étudier le passage de l'énergie libre à l'énergie liée selon une organisation complexe et hiérarchisée. Cette hétérogénéité est le fruit des premières constructions de la psyché dont émerge, tout d'abord, selon Solms, des formes primaires de conscience essentiellement de nature affective et qui

\footnotetext{
${ }^{15}$ C'est là d'ailleurs un point technique qui différencie sur le plan ontologique le clinicien d'orientation psychanalytique des pratiques plus opératoires, en particulier dans le champ médical, dans lesquelles, à l'inverse, on cherchera à diminuer autant que faire se peut tout effet de surprise.
} 
correspondent à une associativité débridée. La fonction principale du psychisme sera, secondairement, de former une représentation cohérente du monde qui limite les effets de désorganisation de la réalité psychique. Mais cela nécessite une conscience secondarisée qui obéit à d'autres logiques associatives que celles des structures plus archaïques. L'articulation entre ces différents niveaux d'associativité se ferait notamment par le biais du mode par défaut, équivalent neurobiologique du Moi, dont la fonction essentielle serait un travail d'intégration et de structuration de logiques psychiques parfois opposées. Le cadre thérapeutique permettra de relancer ces processus d'articulation et d'intégration, en particulier lorsque des éléments provenant de la rencontre avec l'environnement ont été clivés de la subjectivité.

Pour conclure, nous souhaiterions évoquer deux perspectives qui permettent d'approfondir les pistes ouvertes dans ce travail. La première concerne le fait que nous avons essentiellement abordé l'associativité du point de vue de la réalité psychique du patient, mais l'associativité du clinicien mériterait également d'être analysée dans le détail. Ainsi, la théorie elle-même peut être conceptualisée comme un "support» internalisé de l'associativité du clinicien. Les dispositifs théoriques et pratiques (les supervisions, les groupes de lecture, les écoles de pensée, etc.), fruits d'une longue tradition clinique provenant de la «sédimentation » des cadres externalisés antérieurs, favorisent et maintiennent une associativité particulière chez le clinicien. Celui-ci évolue ainsi au sein d'une «trame associative », qui étaye son associativité et favorise, en dernier lieu, celle du patient dans le cadre psychothérapique. Ces logiques correspondent à des «transferts d'associativité » entre les différents « milieux », sur les plans culturels et anthropologiques, ainsi qu'à des conditions de compatibilité ou d'incompatibilité de ces logiques associatives, ce sur quoi l'ethnopsychiatrie s'est déjà longuement penchée.

La deuxième piste qui pourrait être explorée dans la continuité de nos réflexions porte davantage sur le plan cognitif et la manière dont on pourrait tenter de comprendre de façon plus détaillée ce qui fonde le fonctionnement profondément associationniste du psychisme. En somme, pourquoi ce besoin d'un transfert associatif de soi à l'autre, de soi à l'objet et de soi à soi, pour intégrer et métaboliser la matière psychique? Ces différents registres sont conceptualisables comme représentant un ensemble de systèmes complexes qui interagissent entre eux tout en se différenciant progressivement et en affinant leurs interrelations réciproques. Une forme «d'associativité primordiale » se cache probablement aux fondements même de ce processus que nous pourrions éclairer à partir des recherches sur la synesthésie. Celle-ci, issue du terme grec «sunaisthêsis »- signifiant «perception simultanée »-, désigne l'association apparemment inappropriée de plusieurs sens. Par exemple, une personne percevra visuellement, de manière colorée et hallucinatoire, des notes de musiques ou des lettres de l'alphabet. Il se produit alors un transfert automatique et immédiat d'une modalité sensorielle à une autre. Le développement cérébral nécessite une inhibition de cette première forme d'associativité [67] mais certains sujets héritent néanmoins de vestiges partiels de cette associativité débridée - notamment des patients autistes [43]- qui, parfois, refont soudainement surface à la faveur d'un événement de vie ou d'un état de conscience particulier. Cette associativité primordiale serait présente en toile de fond du psychisme et la transmodalité symbolisante en serait, le «reste», le «vestige». Des recherches innovantes associant, paradigmes cliniques et cognitifs, notamment dans le champ de l'imagerie à partir de sujets rapportant des phénomènes de synesthésie, permettraient peut-être d'affiner notre compréhension de ces logiques associatives et symbolisantes. 


\section{Références}

[1] Ansermet F, Magistretti P. À chacun son cerveau : plasticité neuronale et inconscient. Paris: Odile Jacob; 2004.

[2] Bion WR. Aux sources de l'expérience, $2003^{\mathrm{e}}$ éd., Paris: PUF; 1962.

[3] Botella C. Rêverie-reverie et travail de figurabilité. Table ronde, débats sans frontières. Paris: Société Psychanalytique de Paris; 2006.

[4] Botella C, Botella S. La problématique de la régression formelle de la pensée et de l'hallucinatoire. La psychanalyse : questions pour demain. Paris: PUF; 1990.

[5] Botella C, Botella S. La figurabilité psychique, $2007^{\circledR}$ éd., Paris: In press; 2001.

[6] Buckner RL, Andrews-Hanna JR, Schacter DL. The brain's default network: anatomy, function, and relevance to disease. Ann N Y Acad Sci 2008;1124:1-38.

[7] Cardeña E, Winkelman M. Altering consciousness: multidisciplinary perspectives. London: Praeger; 2011.

[8] Carhart-Harris RL, Friston KJ. The default-mode, ego-functions and free-energy: a neurobiological account of Freudian ideas. Brain 2010;133(4):1265-83.

[9] Carhart-Harris RL, Leech R, Hellyer PJ, Shanahan M, Feilding A, Tagliazucchi E, et al. The entropic brain: a theory of conscious states informed by neuroim- aging research with psychedelic drugs. Front Hum Neurosci 2014;8:20.

[10] Damasio AR. L'autre moi-même. Les nouvelles cartes du cerveau, de la conscience et des émotions. Paris: O. Jacob; 2010.

[11] David C. La bisexualité psychique. Paris: Payot; 1992.

[12] Dienes Z. Bayesian versus orthodox statistics: which side are you on? Perspect Psychol Sci 2011;6(3):274-90.

[13] Donnet J-L. Le procédé et la règle : l'association libre analytique. Rev Fr Psychanal 2012;76(3):695-723.

[14] Edelman G. Biologie de la conscience. Paris: Odile Jacob; 2000.

[15] Eschel O. Where are you, my beloved? On absence, loss, and the enigma of telepathic dreams. Int J Psychoanal 2006;87(6):1603-27.

[16] Evans JSBT. In two minds: dual-process accounts of reasoning. Trends Cogn Sci 2003;7(10):454-9.

[17] Evrard R. Les expériences réputées psychotiques dans la population générale. Ann Med Psychol 2011;160:582-7.

[18] Evrard R, Rabeyron T. Les psychanalystes et le transfert de pensée : enjeux historiques et actuels. Evol Psychiatr (Paris) 2012;77(4):589-98.

[19] Freud S. 2010 éd., Constructions dans l'analyse. Euvres complètes, XX, 2010 éd. Paris: PUF; 1937.

[20] Freud S. La méthode d'interprétation des rêves. L'interprétation des rêves. Paris: PUF; 1900.

[21] Freud S. Cinq psychanalyses, $2008^{\mathrm{e}}$ éd., Paris: PUF; 1909.

[22] Freud S. L'Homme aux rats : un cas de névrose obsessionnelle, $2010^{\mathrm{e}}$ éd., Paris: Payot; 1907.

[23] Freud S. La technique psychanalytique, $1981^{\mathrm{e}}$ éd., Paris: PUF; 1919.

[24] Freud S. Esquisse d'une psychologie scientifique, $1956^{\mathrm{e}}$ éd., Paris: PUF; 1895.

[25] Freud S. $1988^{\mathrm{e}}$ éd., L'inconscient. CEuvres complètes, XIII, $1988^{\mathrm{e} e ́ d . ~ P a r i s: ~ P U F ; ~} 1915$.

[26] Freud S. Remémorer, répéter, perlaborer. La technique psychanalytique, $1970^{\mathrm{e}}$ éd., Paris: PUF; 1914.

[27] Freud S. L'Analyse finie et l'analyse infinie. Euvres complètes, XX. Paris: PUF; 1937.

[28] Freud S. Malaise dans la culture. Paris: PUF; 1930.

[29] Freud S, Breuer J. Études sur l'hystérie, 2002e éd., Paris: PUF; 1895. 
[30] Friston K. The free-energy principle: a rough guide to the brain? Trends Cogn Sci 2009;13(7):293-301.

[31] Gay MC. Y a-t-il un lien entre hypnotisabilité et psychopathologie ? Ann Med Psychol Rev Psychiatr 2005;163(2):127-31.

[32] Georgieff N. Psychanalyse, neurosciences et subjectivités. Neuropsychiatr Enfance Adolesc 2010;58(6-7):343-50.

[33] Golse B. Entre neurosciences et psychanalyse. Adolescence 2012;80(2): 269-285.

[34] Green A. Note sur les processus tertiaires. Rev Fr Psychanal 1995;151-5.

[35] Haley J, Robert F. Un thérapeute hors du commun : Milton H. Erickson. Paris: Desclée de Brouwer; 1995.

[36] Kahneman D. A perspective on judgment and choice: mapping bounded rationality. Am Psychol 2003;58(9):697-720.

[37] Kahneman D. Thinking, fast and slow. New York: Farrar, Straud and Giroux; 2011.

[38] Koch C. À la recherche de la conscience : une enquête neurobiologique. Paris: Odile Jacob; 2006.

[39] Laplanche J, Pontalis J-B. Vocabulaire de la psychanalyse, 2002e e' d., Paris: PUF; 1967.

[40] Magistretti P, Ansermet F. Neurosciences et psychanalyse. Paris: Odile Jacob; 2010.

[41] Méheust B. Somnambulisme et médiumnité. Paris: Les Empêcheurs de penser en rond; 1999.

[42] De M'Uzan M. La bouche de l'inconscient. Paris: Gallimard; 1994.

[43] Neufeld J, Roy M, Zapf A, Sinke C, Emrich HM, Prox-Vagedes V, et al. Is synesthesia more common in patients with Asperger syndrome? Front Hum Neurosci 2013;7.

[44] Panksepp J. Affective neuroscience: the foundations of human and animal emotions. Oxford: Oxford university press; 1998.

[45] Parat C. L'affect partagé. Rev Fr Psychosom 2013;44(2):167-82.

[46] Poenaru L. L'hallucinatoire freudien et les neurosciences : pour un cadre interdisciplinaire. Rev Fr Psychanal 2013;77(2):555-73.

[47] Rabeyron T. Les processus de symbolisation et de représentation comme espace transitionnel pour la psychanalyse et les neurosciences. Evol Psychiatr (Paris) 2014. http://dx.doi.org/10.1016/j.evopsy.2015.03.003.

[48] Rabeyron T. Les expériences exceptionnelles : entre neurosciences et psychanalyse. Rech En Psychanal 2009;8:282-96.

[49] Rabeyron T, Chouvier B, Le Maléfan P. Clinique des expériences exceptionnelles : du trauma à la solution paranormale. Evol Psychiatr (Paris) 2010;75(4):633-53.

[50] Raichle ME, Snyder AZ. A default mode of brain function: a brief history of an evolving idea. Neuroimage 2007;37(4):1083-90.

[51] Reik T. Le psychologue surpris : deviner et comprendre les processus inconscients, $2001^{\mathrm{e}}$ éd., Paris: Denoë 1; 1935.

[52] Roussillon R. Du baquet de Mesmer au baquet de Sigmund Freud. Paris: PUF; 1992.

[53] Roussillon R. L'associativité. Libr Cah Psychanal 2009;20(2):19-35.

[54] Roussillon R. L'associativité polymorphique et les extensions de la psychanalyse. Carnet Psy 2012;162(4):27-31.

[55] Roussillon R. La disposition d'esprit clinique. Manuel de pratique clinique. Paris: Elsevier; 2011.

[56] Roussillon R. Agonie, clivage et symbolisation. Paris: PUF; 1999.

[57] Roussillon R. Le plaisir et la répétition : théorie du processus psychique. Paris: Dunod; 2001.

[58] Roussillon R, Chabert C, Ciccone A, Ferrant A. Manuel de psychologie et psychopathologie clinique générale. Paris: Masson; 2007. 
[59] Roustang F. Savoir attendre pour que la vie change. Paris: Odile Jacob; 2006.

[60] Solms M. The conscious id. Neuropsychoanalysis 2013;15(1):5-19.

[61] Solms M, Turnbull O. What is neuropsychoanalysis? Neuropsychoanalysis Interdiscip J Psychoanal Neurosci 2011;13(2):133-46.

[62] Stern A. Investigation psychanalytique sur le groupe borderline des névroses. Quelle thérapie engager? Rev Fr Psychanal 2011;75(2):331-48.

[63] Stern D. Le monde interpersonnel du nourrisson, 2006 éd., Paris: PUF; 1989.

[64] Stern D-N. Le moment présent en psychothérapie : un monde dans un grain de sable. Paris: Odile Jacob; 2003.

[65] Tellier I, Finkel A. Cognitive style of decision making narrations. The cognitive level. Endres-Niggemeyer. Duisburg: LAUD series of Cognitive Linguistics; 1995. p. 41-59.

[66] Varela F. Invitation aux sciences cognitives, 1996 éd., Paris: Le seuil; 1988.

[67] Ward J. Synesthesia. Annu Rev Psychol 2013;64(1):49-75.

[68] Widlöcher D. Les nouvelles cartes de la psychanalyse. Paris: Odile Jacob; 1996.

[69] Wilson SC, Barber TX. The fantasy-prone personality: implications for understanding imagery, hypnosis, and parapsychological phenomena. Imagery: current theory, research, and application: current theory, research and application. New York: Wiley; 1983.

[70] Winnicott DW. La crainte de l'effondrement et autres situations cliniques, 2000e éd., Paris: Gallimard; 1989.

[71] Winnicott DW. De la pédiatrie à la psychanalyse. Psychose et soins maternels. Paris: Payot; 1989. 